\title{
Flexible Ureterorenoscopy Versus Extracorporeal Shock Wave Lithotripsy for the Treatment of Renal Pelvis Stones of 10-20 mm in Obese Patients
}

\author{
Babak Javanmard, Mohammad Reza Razaghi, Anahita Ansari Jafari*, Mohammad Mohsen Mazloomfard \\ Laser Application in Medical Sciences Research Center, Shahid Beheshti University of Medical Sciences, Tehran, \\ Iran
}

*Correspondence to

Anahita Ansari Jafari, M.D; Laser Application in Medical Sciences Research Center, ShahidBeheshti University of Medical Sciences, Tehran, Iran.

Tel: +98- 2122718021

Fax: $+98-2122749221$

Email: mazloomfard@yahoo.com

Published online 27 October 2015

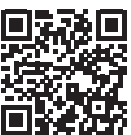

\begin{abstract}
Introduction: To compare outcomes of retrograde intrarenal surgery (RIRS) with extracorporeal shock wave lithotripsy (ESWL) as treatment of choice.

Methods: A total number of 46 patients with renal pelvic stones $10-20 \mathrm{~mm}$ and body mass index $(\mathrm{BMI})>30$ randomized in two groups underwent RIRS and ESWL from 2011 to 2014 and followed for 3 months.

Results: The patients mean \pm SD age was $36.1 \pm 13.1$ years in ESWL and $33.2 \pm 11.4$ years in RIRS groups $(P=.1)$ with comparable BMI in both groups (36.2 vs 38.1). In ESWL and RIRS groups, the operation time was $72.2 \pm 21$ vs $66.5 \pm 19$ minutes $(P=.061)$, respectively. Stone free rate (SFR) at 3 months was $68 \%$ in ESWL group vs $90.4 \%$ in RIRS group $(P=.019)$. The complication rate was $20 \%$ in ESWL group vs $14.2 \%$ in RIRS group $(P=.211)$ but all of them were minor and managed conservatively.

Conclusion: According to our study, RIRS procedure in comparison with ESWL is a safe and successful option of treatment for renal pelvis stone of $10-20 \mathrm{~mm}$ in obese people.

Keywords: Lithotripsy; Obesity; Stone, Kidney.
\end{abstract}

\section{Introduction}

Treatment of renal stones has seen significant changes over the last few decades with a shift from open surgery to minimally invasive interventions. The European Association of Urology (EAU), updates and publishes guidelines for urolithiasis and treatment algorithms every year. EAU 2014 guidelines state that stones between $10-20 \mathrm{~mm}$ in every location should be treated with extracorporeal shock wave lithotripsy (ESWL) or endourologic interventions. ${ }^{1}$ The efficacy of ESWL drops significantly for large renal stones. Other factors such as stone composition, position, kidney malposition and obesity of patient have negative effects on the success rate of ESWL. ${ }^{1}$ Obesity is now considered a worldwide epidemic, affecting >300 million people, more obese patients are presenting with urinary calculi. Because of the increased distance from the skin to the stone, ESWL might not be successful in obese patients. ${ }^{2}$ With advances in the designs of ureterorenoscopes (URSs), especially the introduction of Holmium:YAG (yttrium aluminum garnet) laser into the market and worldwide accepted use of this laser during URS, most of kidney stones can nowadays be managed by flexible ureterorenoscopy (F-URS). ${ }^{3}$ It seems that obesity do not affect the results of F-URS in contrast to ESWL. This clinical trial was conducted to compare the results and complications of ESWL with RIRS in management of renal pelvis stone $10-20 \mathrm{~mm}$ in obese patients (BMI $>30)$.

\section{Methods}

Study Population

Between March 2010 and March 2014, a total number of 58 patients with renal pelvic stones $10-20 \mathrm{~mm}$ and $\mathrm{BMI}>30$ and were candidates for treatment in our urology center enrolled in the study after a routine preoperative evaluation. The inclusion criteria were renal pelvic stones $10-20 \mathrm{~mm}$ in diameter and BMI $>30$. The exclusion criteria were kidney anomalies, uncontrolled coagulopathies, positive urinary culture, ureteral obstruction, pregnancy, and renal failure (serum creatinine $\geq 3 \mathrm{mg} / \mathrm{dl}$ ) and history of failed previous procedure for treatment of stone.

The nature of the study was explained to each patient and informed consent was obtained. The design of this research was approved by the ethics committee of the laser application in medical sciences research center (LAMSRC).

The preoperative evaluation consisted of tests such as urine analysis, urine culture, and renal function tests. Before procedures, urine cultures were obtained, and if positive, antibiotics were administered. Computed tomography (CT) scan was the primary modality to determine the size, number and location of calculi, and the anatomy of

Please cite this article as follows: Javanmard B, Razaghi MR, Ansari Jafari A, Mazloomfard MM. Flexible ureterorenoscopy versus extracorporeal shock wave lithotripsy for the treatment of renal pelvis stones of 10-20 mm in obese patients. J Lasers Med Sci. 2015;6(4):162166. doi:10.15171/jlms.2015.12. 
the upper urinary tract. Prophylactic intravenous antibiotics were administered before surgery.

\section{Study Design}

This was a single center, parallel-group study conducted in the urology department of Tajrish hospital in Tehran, Iran. Patients were randomly assigned to one of two groups according to the method of treatment: ESWL (group 1) and RIRS (group 2) (25 patients in group 1 and 21 patients in group 2). Simple randomization was carried out using computerized random numbers. Sample size was determined after consideration of type 1 statistical error $<5 \%$; and type 2 statistical error $<20 \%$.

\section{Surgical Technique}

For patients in group 1 all of the procedures were performed by an experienced urologist on ESWL therapy and a technician with Dornier Lithotripter (Dornier MedTech, Wessling, Germany). An intravenous sedative anesthesia was administered before the sessions. Stone location was identified with the aid of fluoroscopy/ultrasound guidance. A maximum of 3000 shocks were applied at 80 shocks per minute during each session or until complete disintegration of the stones were observed.

In group 2, the procedure was performed under spinal anesthesia while in lithotomy position by a surgeon. After inserting a semirigid ureteroscope under endovision guidance through the bladder, a 0.035 -inch hydrophilic coated guide-wire was introduced through the channel into the ureteral orifice and then ureteroscopy was performed with hydrodilation to dilate the ureter. Thereafter, an 11 Fr ureteral access sheath was placed. A 4 Fr or 6 Fr feeding tube was placed transurethrally to maintain low pressure of the bladder. The 8.5/5.3 Fr flexible ureteroscope (Olympus) was introduced under fluoroscopic guidance up to the renal pelvis until the stone was identified. Stone fragmentation was performed using a Holmium:YAG laser (manufacture in Iran) with $200 \mathrm{~mm}$ fibers. When fragmentation was complete, final ureteronephroscopy followed by a control fluoroscopy were carried out for any residual stone detection. JJ stent was placed in the ureter for 2 weeks in cases of difficult dilation, prolonged procedure or residual stone. If no ureteral injury occurred, a ureteral stent was inserted and fixed to the Foley catheter. The ureteral catheter was removed the day after the procedure.

\section{Outcome Assessment}

Due to the difference between the two methods, it was not possible to blind the surgical team from knowledge of which procedure a participant received. Stone-free status as the primary parameters used to evaluate efficacy and residual stone burden were determined by abdominal CT scan 3 months after treatment.

The procedure was considered as a failure in stone residual fragments $\geq 3 \mathrm{~mm}$ detected by these studies. If there was any residual stone detected at 3-month's work-up, the second session of treatment was performed.
Demographic and stones characteristics, stone free rate (SFR) in 3 months after procedure, time of operation (from the first to last shock in ESWL and from the ureteroscopy insertion to Foley insertion in RIRS), and complications such as bleeding, urosepsis and collecting system perforation were compared between the 2 groups.

Data analysis was performed using SPSS version 16.0 software (SPSS Inc., Chicago, IL, USA) using student's $t$ test and chi-square test. A $P<.05$ was considered statistically significant.

\section{Results}

A total of 46 patients with renal pelvic calculi and BMI greater than 30 were included in the present study and were classified into those who had SWL $(n=25)$ and F-URS $(n=21)$. The demographics of both groups were similar with respect to age, sex distribution, comorbidities (hypertension [HTN] and diabetes mellitus [DM]), side of stone, mean size of stones and BMI (Table 1).

Treatment and post-treatment data are summarized in Table 2. The retreatment rate was significantly higher in the ESWL group because of the need for multiple sessions in $36 \%$ of patients. For F-URS patients, a second session of F-URS was needed for 2 patients. In these 2 patients, because of ureteral kinking and obstruction, insertion of access sheat failed and a JJ stent was fixed and then F-URS was performed after 2 weeks.

Mean operation duration of all sessions of treatment was shorter with F-URS than ESWL group $(72.2 \pm 21 \mathrm{~min}$ vs $66.5 \pm 19 \mathrm{~min}, P=.061)$ but this difference was not significant. The 3-month SFR was significantly better after F-URS ( $90.4 \%$ vs $68 \%$ for ESWL, $P=.019)$.

Table 1. Demographic and Clinical Characteristics

\begin{tabular}{llll}
\hline & Group 1 (ESWL) & Group 2 (F-URS) & P Value \\
\hline Age, mean (SD) & $36.1(13.1)$ & $33.2(11.4)$ & .109 \\
Gender, No. (\%) & $\begin{array}{l}\text { Male, 15 (60) } \\
\text { Female, 10 (40) }\end{array}$ & Male, 13 (61) & .67 \\
Side of stone, & Rt, 13 (52) & Rt, 12 (57.1) & .98 \\
No. (\%) & Lt, 12 (48) & Lt, 9 (42.8) & \\
BMI, mean(SD) & $36.2(3.8)$ & $38.1(3.2)$ & .073 \\
DM & 4 & 3 & .31 \\
HTN & 5 & 3 & .27 \\
Stone size, & $16.3(2.4)$ & $17.1(1.9)$ & .88 \\
Mean (SD) & & & \\
\hline
\end{tabular}

Abbreviations: HTN, hypertension; DM, diabetes mellitus; BMI, body mass index; ESWL, extracorporeal shock wave lithotripsy; F-URS, flexible ureterorenoscopy.

Table 2. Treatment and Posttreatment Data

\begin{tabular}{llll}
\hline & $\begin{array}{l}\text { Group 1 } \\
\text { (ESWL) }\end{array}$ & $\begin{array}{l}\text { Group 2 } \\
\text { (F-URS) }\end{array}$ & P Value \\
\hline Retreatment rate, No. (\%) & $11(36 \%)$ & $2(9.5 \%)$ & $<.001$ \\
Complication, No. (\%) & $5(20 \%)$ & $3(14.2 \%)$ & .211 \\
SFR 3 months, No. (\%) & $17(68 \%)$ & $19(90.4 \%)$ & $<.019$ \\
Operation time, mean (SD) & $72.2(21)$ & $66.5(19)$ & $<.061$ \\
\hline
\end{tabular}

Abbreviations: ESWL, extracorporeal shock wave lithotripsy; F-URS, flexible ureterorenoscopy; SFR, stone free rate. 
The incidence of complications was higher in ESWL patients (20\% vs $14.2 \%$ for F-URS), but the difference was not statistically significant $(P=.211)$. One intraoperative complication was encountered due to perforation of the upper ureter. It was successfully treated by a JJ stent for 6 weeks. Postoperative fever developed in 2 patients after F-URS and could be successfully treated with antibiotics and antipyretics. Complications after ESWL included obstructing steinstrasse in 2 patients and fever in two. One patient was hospitalized for gross hematuria and successfully controlled with medical treatment.

\section{Discussion}

Urinary stone treatment improvements over the recent years allowed endoscopic procedures to be performed in almost any situation. The incidence of urolithiasis varies in the literature affecting $5 \%-15 \%$ of the population worldwide. ${ }^{4}$ The parallel growth in prevalence of obesity and morbid obesity over the past decades has increased the management of stone disease in this population. ${ }^{5,6}$ The association between urolithiasis and obesity is established in the literature and has shown that weight gain increases the urinary excretion of substances such as oxalate and uric acid, leading to stone formation. ${ }^{7,8}$ Studies also indicate that obese patients suffer from urolithiasis twice as much as the non-obese and recurrence rates are close to $50 \% .{ }^{9}$ Conversely, due to conflicting data and the development of new endoscopic instruments the optimal management of stone disease in these patients is still in discussion.

EAU 2014 guidelines advices ESWL as the first line treatment alternative for stones $<2 \mathrm{~cm}$ located in the renal pelvis, upper or middle calices. ${ }^{10}$ In ESWL stone clearance depends on a number of factors such as stone related (burden, location, and density), renal related (anatomical features) and pertinently patient related (BMI, muscle-skeletal deformity, and social security disability [SSD] ) characteristics. Pareek et al demonstrated that BMI independently predicted the SWL outcome. They suggested that patients with a $\mathrm{BMI} \geq 30 \mathrm{~kg} / \mathrm{m}^{2}$ would be more suitably treated by endoscopic manipulation. ${ }^{11}$ In another study by the same author, SSD of $12 \mathrm{~cm}$ was an independent predictor of unfavorable outcomes. ${ }^{12}$ Further, SWL may also produce detrimental side effects such as HTN and DM, which are already risk factors associated with obesity. Between 21\% and 70\% of clinically insignificant residual fragments post-SWL can regrow, ${ }^{13,14}$ potentially causing complications that require further intervention in more than half of these cases. ${ }^{15}$

Improvements in endoscopic technology have made retrograde stone removal more attractive. ${ }^{16}$ In fact, endourologic procedures expand their role continuously for treatment of urinary calculi. Furthermore, ureteroscopy does not appear to be affected by body habitus (for example morbid obese, sever scoliosis, discopathy, etc). In a retrospective, single institution study, the SFRs were similar between 107 obese patients and non-obese patients. ${ }^{17}$ Another recent study published by Best et $\mathrm{al}^{18}$ showed statistically equivalent SFR in obese (91\%) and non-obese (81\%) patients who underwent flexible URS for proximal ureteral stones. Thus, the improved success rates of URS compared to SWL may promote this approach as the method of choice to treat our increasingly obese population. More recently, Delorme et $\mathrm{al}^{19}$ analyzed the feasibility of flexible URS in obese patients and found equivalency in terms of operative time, rate of complications and overall SFR compared to non-obese patients. A contemporary meta-analysis published by Aboumarzouk et $\mathrm{al}^{20}$ regarding the feasibility of URS in obese patients showed good outcomes with a SFR of $87.5 \%$.

Successful ESWL treatment in obese patients was reported with an overall SFR at 3 months of $68 \%$ compared to $80 \%-85 \%$ in non-obese patients. ${ }^{21}$ We achieved an overall SFR of $68 \%$ in patients who underwent ESWL and it was similar with the results in the literature.

According to a recent meta-analysis the use of RIRS on obese patients is efficient, with an overall stone-free rate of $87.5 \%$, and minor complication rate (grade I, II, or III) of $11.4 \% .^{20}$

In our series SFR at 3 months was $90.4 \%$ and complication rate $14.2 \%$. According to our experience in referral center of laser treatment of stone, obese patients could easily be managed with RIRS procedure with acceptable stone clearance. After each session of ESWL, beside the fact that residual calculi that remain within the kidney can lead to recurrent stone formation ${ }^{22}$; stone fragments expulsion is always followed by renal colic. ${ }^{23}$ Actually, repeated treatment sessions of ESWL induced multiple episodes of pain in patients. High success rate at first session of RIRS makes it favorable for individual whom cannot tolerate aching. Steinstrasse formation can also cause discomfort in patients and was reported in $2 \%$ to $10 \%$ of ESWL cases. ${ }^{24}$ This complication that directly correlates with increasing stone burden was diagnosed in $4 \%$ (2\25) of the patients in ESWL group of our study. Steinstrasse was one of the drawbacks of ESWL which increases the number of auxiliary treatments in patients. Despite a lower SFR and higher retreatment rates, ESWL remains a treatment option because of its non-invasive nature and high level of acceptance by physicians and patients.

The most important results of this comparison were the significantly higher SFR and lower retreatment rate after F-URS, while complications were not significantly more in the F-URS group (Table 2). All complications could be treated with either medical treatment or endoscopic techniques. Improvements in the techniques and instruments, such as digital F-URS and ureteric access sheath can help increase the safety and efficacy of F-URS in the treatment of renal stone. ${ }^{25}$ The access sheath allows free drainage of irrigation from the kidney, thus improving vision, and prevents potentially harmful elevations in intrarenal pressure. It has the potential to improve SFRs by allowing passive or active retrieval of fragments as the ureteroscope can be introduced many times through the sheath over a short period of time. Despite the advantages of ureteral access sheaths in facilitating multiple passages of the flex- 
ible ureteroscope, providing lower intrarenal pressures, and better flow through the ureteroscope, it might be associated with ureteral injuries and ureteral strictures. ${ }^{26}$ In a study by Traxer and Thomas, ${ }^{27}$ the incidence of injuries caused by ureteral access sheaths during RIRS was $46.5 \%$. For this reason, routine ureteral stenting is often recommended if a ureteral access sheath is used. In our study, ureteral stent or JJ stent was inserted for all patients of RIRS group. In order to omit the extra admission for JJ stent removal, we preferred to introduce ureteral stent unless the case was complicated or failed.

One drawback of RIRS is its long operation time that is dependent to stone size, number, location and experience of the surgeon. In our study because of the location of the stones (renal pelvis) and our experience in laser ablation, the operation time was not significantly different between 2 groups. Another disadvantage of F-URS included the need for expensive instruments such as fragile ureteroscopes, a laser machine and tipless nitinol baskets. It is therefore advised to have a standby flexible ureteroscope and to handle it with great care during the operation as well as during cleaning and sterilization. In addition our laser machine was made in Iran and its maintenance costs were low for our research center. Although, we did not investigate ESWL and F-URS in terms of costs.

There are several limitations of our study. First of all, our study had a small sample size. Postoperative or post-procedure pain scores, analgesic need and stone composition of the patients which can significantly affect the outcomes of the procedures were not evaluated in our study. Also we did not perform and compare a cost-effectiveness analysis for F-URS and ESWL.

Equipment required for RIRS including flexible ureteroscope and holmium laser are expensive and not available in many centers. ${ }^{28}$ Also, unfamiliarity of urologists with this technique and low level of experience result in RIRS being performed in limited referral centers. According to our study, RIRS procedure is a safe and successful option of treatment for renal stone of $\leq 2 \mathrm{~cm}$ in obese patients. It seems that in a near future, use of this technique will be outspread in many centers. According to our study, for treatment of renal pelvic stones $10-20 \mathrm{~mm}$ in length in obese patients (BMI $>30$ ), F-URS provided a significantly higher SFR and lower retreatment rate compared with ESWL. The incidence of complications after F-URS was not significantly different from ESWL and the severity of complications was comparable. These results support the increasing role of F-URS in the treatment of renal pelvic stones $10-20 \mathrm{~mm}$ in length in obese patients (BMI >30).

\section{Conclusion}

According to our study, RIRS procedure in comparison with ESWL is a safe and successful option of treatment for renal pelvic stones of 10 to $20 \mathrm{~mm}$ in obese patients.

\section{Conflict of Interest}

The authors have no conflict of interest to declare.

\section{References}

1. Abe T, Akakura K, Kawaguchi M, et al. Outcomes of shockwave lithotripsy for upper urinary-tract stones: a large-scale study at a single institution. J Endourol. 2005;19:768-777. doi:10.1089/end.2005.19.768

2. Afane JS, Olweny EO, Bercowsky E, et al. Flexible ureteroscopes: a single center evaluation of the durability and function of the new endoscopes smaller than 9Fr. J Urol. 2000;164:1164-1168. doi:10.1016/ s0022-5347(05)67133-9

3. Bas O, Bakirtas H, Sener NC, et al. Comparison of shock wave lithotripsy, flexible ureterorenoscopy and percutaneous nephrolithotripsy on moderate size renal pelvis stones. Urolithiasis. 2014;42(2):115-120. doi:10.1007/s00240-013-0615-2.

4. Moe OW. Kidney stones: pathophysiology and medical management. Lancet. 2006;367:333-344. doi:10.1016/s0140-6736(06)68071-9

5. Stamatelou KK, Francis ME, Jones CA, Nyberg LM, Curhan GC. Time trends in reported prevalence of kidney stones in the United States: 1976-1994. Kidney Int. 2003;63:1817-1823. doi:10.1046/j.15231755.2003.00917.x

6. Ogden CL, Carroll MD, Curtin LR, McDowell MA, Tabak CJ, Flegal KM. Prevalence of overweight and obesity in the United States, 1999-2004. JAMA. 2006; 295:1549-1555. doi:10.1001/jama.295.13.1549

7. Powell CR, Stoller ML, Schwartz BF, et al. Impact of body weight on urinary electrolytes in urinary stone formers. Urology. 2000;55:825-30. doi:10.1016/s00904295(99)00617-2

8. Negri AL, Spivacow FR, Del Valle EE, Forrester M, Rosende G, Pinduli I. Role of overweight and obesity on the urinary excretion of promoters and inhibitors of stone formation in stone formers. Urol Res. 2008;36:303-307. doi:10.1007/s00240-008-0161-5

9. Sutherland JW, Parks JH, Coe FL. Recurrence after a single renal stone in a community practice. Miner Electrolyte Metab.1985;11:267-269.

10. Türk C, Knoll T, Petrik A, et al. EuropeanAssociation of Urology, Guidelines on urolithiasis. Eur Urol. 2015. pii: S0302-2838(15)00700-9.

11. Pareek G, Armenakas NA, Panagopoulos G, Bruno JJ, FracchiaJá. Extracorporeal shock wave lithotripsy success based on body mass index and Hounsfield units. Urology. 2005;65:33-36. doi:10.1016/j. urology.2004.08.004

12. Pareek G, Hedican SP, Lee FT Jr, Nakada SY. Shock wave lithotripsy success determined by skin-tostone distance on computed tomography. Urology. 2005;66:941-944. doi:10.1016/j.urology.2005.05.011

13. Beck EM, Riehle RA Jr. The fate of residual fragments after extracorporeal shock wave lithotripsy monotherapy of infection stones. J Urol. 1991;145:69.

14. Yu CC, Lee YH, Huang JK, et al. Long-term stone regrowth and recurrence rates after extracorporeal shock wave lithotripsy. Br J Urol. 1993;72:688-691. 
15. Khaitan A, Gupta NP, Hemal AK, Dogra PN, Seth A, Aron M. Post-ESWL, clinically insignificant residual stones: reality or myth? Urology. 2002;59:20-24. doi:10.1016/s0090-4295(01)01494-7

16. El-Hout Y, Elnaeema A, Farhat WA. Current status of retrograde intrarenal surgery for management of nephrolithiasis in children. Indian J Urol. 2010;26(4):568-572. doi:10.4103/0970-1591.74463

17. Natalin R, Xavier K, Okeke Z, Gupta M. Impact of obesity on ureteroscopic laser lithotripsy of urinary tract calculi. Int Braz J Urol. 2009;35:36-41. doi:10.1590/s1677-55382009000100006

18. Best SL, Nakada SY. Flexible ureteroscopy is effective for proximal ureteral stones in both obese and nonobese patients: a two-year, single-surgeon experience. Urology. 2011;77:36-39. doi:10.1016/j. urology.2010.05.001

19. Delorme G, Huu YN, Lillaz J, et al. Ureterorenoscopy with holmium-yttrium-aluminum-garnet fragmentation is a safe and efficient technique for stone treatmentin patients with a body mass index superior to $30 \mathrm{~kg} / \mathrm{m} 2$. J Endourol. 2012;26:239-43. doi:10.1089/ end.2011.0391

20. Aboumarzouk OM, Somani B, Monga M. Safety and efficacy of ureteroscopic lithotripsy for stone disease in obese patients: a systematic review of the literature. BJU Int. 2012; 110(8 Pt B):E374-E380. doi:10.1111/ j.1464-410x.2012.11086.x

21. Peterson RF, Lifshitz DA, Kuo RL, Siqueira TM Jr, Lingeman JE. Shock wave lithotripsy monotherapy for renal calculi. Int Braz J Urol. 2002;28:291-301.
22. Fine JK, Pak CY, Preminger GM. Effect of medical management and residual fragments on recurrent stone formation following shock wave lithotripsy. J Urol. 1995;153(1):27-32. doi:10.1097/00005392199501000-00010

23. Gravina GL, Costa AM, Ronchi P, et al. Tamsulosin treatment increases clinical success rate of single extracorporeal shock wave lithotripsy of renal stones. Urology. 2005;66(1):24-28. doi:10.1016/j. urology.2005.01.013

24. Al-Awadi KA, Abdul Halim H, Kehinde EO, AlTawheed A. Steinstrasse: a comparison of incidence with and without J stenting and the effect of J stenting on subsequent management. BJU Int. 1999;84(6):618621. doi:10.1046/j.1464-410x.1999.00280.x

25. Andonian S, Okeke Z, Smith AD. Digital ureteroscopy: the next step. J Endourol. 2008;22:603-606.

26. Torricelli FC, De S, Hinck B, Noble M, Monga M. Flexible ureteroscopy with a ureteral access sheath: when to stent? Urology. 2014;83(2):278-281.

27. Traxer O, Thomas A. Prospective evaluation and classification of ureteral wall injuries resulting from insertion of a ureteral access sheath during retrograde intrarenal surgery. J Urol. 2013;189:580584. doi:10.1016/j.juro.2012.08.197

28. Mokhless IA, Abdeldaeim HM, Saad A, Zahran AR. Retrograde intrarenal surgery monotherapy versus shock wave lithotripsy for stones 10 to $20 \mathrm{~mm}$ in preschool children: a prospective, randomized study. J Urol. 2014;191(5 Suppl):1496-1499. doi:10.1016/j. juro.2013.08.079 